\title{
Time-Dependent B-Spline R-Matrix Approach to Double Ionization of Atoms by XUV Laser Pulses
}

\author{
Xiaoxu Guan ${ }^{\dagger, 1}$, O Zatsarinny ${ }^{\dagger, 2}$, C J Noble ${ }^{\dagger, \S, 3}, \mathbf{K}$ Bartschat $^{\dagger, 4}$, and \\ B I Schneider ${ }^{\ddagger}, 5$ \\ ${ }^{\dagger}$ Department of Physics and Astronomy, Drake University, Des Moines, IA 50311, USA \\ ${ }^{\S}$ Computational Science and Engineering Department, Daresbury Laboratory, Warrington \\ WA4 4AD, UK \\ ${ }^{\ddagger}$ Physics Division, National Science Foundation, Arlington, Virgina 22230, USA \\ E-mail: ${ }^{1}$ xiaoxu.guan@drake.edu, ${ }^{2}$ oleg.zatsarinny@drake.edu, ${ }^{3}$ cjn@maxnet.co.nz, \\ ${ }^{4}$ klaus.bartschat@drake.edu, ${ }^{5}$ bschneid@nsf.gov
}

Submitted to: J. Phys. B: At. Mol. Opt. Phys.

\begin{abstract}
We present an ab initio and non-perturbative time-dependent approach to the problem of double ionization of a general atom driven by intense XUV laser pulses. After using a highly flexible $B$-Spline $R$-matrix method to generate field-free Hamiltonian and electric dipole matrices, the initial state is propagated in time using an efficient ArnoldiLanczos scheme. Test calculations for double ionization of He by a single laser pulse yield good agreement with benchmark results obtained with other methods. The method is then applied to two-color pump-probe processes, for which momentum and energy distributions of the two outgoing electrons are presented.
\end{abstract}

PACS numbers: $42.50 . \mathrm{Hz}, 32.60 .+\mathrm{i}, 32.80 . \mathrm{Rm}, 32.80 . \mathrm{Fb}$

\section{Introduction}

The two-photon double ionization (DI) of the helium atom induced by intense short XUV laser pulses has received considerable attention from both theorists and experimentalists alike. Instead of listing a large number of references here, we note that much of the recent work was quoted in recent papers [1, 2]. Even within the past few months, however, several additional papers appeared.

Given the intensities and lengths of the laser pulses involved, the numerical approaches used to tackle this problem are all essentially attempts to solve the time-dependent Schrödinger equation (TDSE), beginning with a well-defined initial state before the laser strikes and then propagating this state in the presence of the laser field by one of a number of numerical approaches. Once the laser is switched off, various probabilities and, in some cases, generalized cross sections can be extracted. 
Over the past two years, our group has been working on the development of a general $a b$ initio theoretical approach, which is applicable to complex targets beyond (quasi) twoelectron systems. In two recent papers [3, 4] we outlined how field-free Hamiltonian and electric dipole matrices generated with the highly flexible $B$-Spline $R$-matrix (BSR) [5] suite of codes may be combined with an efficient Arnoldi-Lanczos time propagation scheme to describe the interaction of short intense laser pulses with a complex atom, leading to multiphoton excitation and single ionization. The key points of our method are the following: 1) We employ the BSR code, which allows for the use of non-orthogonal orbital sets, to generate field-free Hamiltonian and dipole matrices. 2) We then set up an efficient ArnoldiLanczos scheme to propagate the initial state in time. 3) Finally, we extract the information by standard projection schemes.

In the present paper, we report on the extension of this approach and the corresponding computer code [6] to allow for two electrons in the continuum and hence the possibility to describe double ionization processes. After outlining the general method, we present a test application to the He problem, for which many benchmark results are available for comparison. Finally, we use the method to investigate pump-probe processes involving two XUV laser pulses whose characteristics, including a time delay, are assumed to be controlled separately in the corresponding experiment.

Unless specified otherwise, atomic units (a.u.) are used throughout this manuscript.

\section{THEORY}

\subsection{The TDBSR Approach}

The ingredients of an appropriate theoretical and computational formulation require an accurate and efficient generation of the Hamiltonian and the electron-field interaction matrix elements, as well as an optimal approach to propagate the TDSE in real time. As mentioned above, there have been numerous calculations for two-electron systems such as He and also $\mathrm{H}_{2}$. While these investigations emphasize the important role of two-electron systems in studying electron-electron correlation in the presence of a strong laser field, in its presumably purest form, experiments with $\mathrm{He}$ atoms are difficult and other noble gases, such as $\mathrm{Ne}$ and Ar, are often favored by the experimental community.

Fully $a b$ initio theoretical approaches, which are applicable to complex targets beyond (quasi) two-electron systems, are still rare. For (infinitely) long interaction times, the $R$ matrix Floquet ansatz [7] has been highly successful. A critical ingredient of this method is the general atomic $R$-matrix method developed over many years by Burke and collaborators in Belfast. A modification of the method, allowing for relatively long though finite-length pulses was described by Plummer and Noble [8]. Recently, a time-dependent formulation [9] of this method was applied to short-pulse laser interactions with Ar [10] and a pump-probe XUV scenario for Ne [11].

Following our recent work on excitation and single-ionization of $\mathrm{Ne}$ [3] and Ar [4], we now describe what needs to be done to extend our approach to double ionization. We start 
with the time-dependent Schrödinger equation

$$
i \frac{\partial}{\partial t} \Psi\left(\boldsymbol{r}_{1}, \ldots, \boldsymbol{r}_{N} ; t\right)=\left[H_{0}\left(\boldsymbol{r}_{1}, \ldots, \boldsymbol{r}_{N}\right)+V\left(\boldsymbol{r}_{1}, \ldots, \boldsymbol{r}_{N} ; t\right)\right] \Psi\left(\boldsymbol{r}_{1}, \ldots, \boldsymbol{r}_{N} ; t\right)
$$

for the $N$-electron wavefunction $\Psi\left(\boldsymbol{r}_{1}, \ldots, \boldsymbol{r}_{N} ; t\right)$, where $H_{0}\left(\boldsymbol{r}_{1}, \ldots, \boldsymbol{r}_{N}\right)$ is the field-free Hamiltonian containing the kinetic energy of the $N$ electrons, their potential energy in the field of the nucleus, and their mutual Coulomb repulsion, while

$$
V\left(\boldsymbol{r}_{1}, \ldots, \boldsymbol{r}_{N} ; t\right)=\sum_{i=1}^{N} \boldsymbol{E}(t) \cdot \boldsymbol{r}_{i}
$$

represents the interaction of the electrons with the laser field $\boldsymbol{E}(t)$ in the dipole length form.

The tasks to be carried out in order to computationally solve this equation and to extract the physical information of interest are:

1. Generate a representation of the field-free Hamiltonian and its eigenstates; these include the initial bound state, other bound states, autoionizing states, as well as singlecontinuum and double-continuum states to represent electron scattering from the residual ion.

2. Generate the dipole matrices to represent the coupling to the laser field.

3. Propagate the initial bound state until some time after the laser field is turned off.

4. Extract the physically relevant information from the final state.

Of particular interest in the experiments mentioned above are processes, in which one, two, or even more electrons undergo significant changes in their quantum state in the presence of an atomic core. These include excitation, single and double ionization, ionization plus simultaneous excitation, or inner-shell ionization with subsequent rearrangement in the hollow ion. The latter processes, in particular, can only be investigated in systems beyond the frequently studied two-electron helium atom or $\mathrm{H}_{2}$ molecule. This generalization to two electrons outside a multi-electron core is far from trivial, but the flexibility of the BSR method is highly advantageous for the tasks 1 and 2. In contrast to many other approaches [12, 13, 14], our method is formulated in a sufficiently general way to be applicable to complex atoms, such as inert gases other than helium and even open-shell systems with non-vanishing spin and orbital angular momenta. In reality, of course, the size of the problems that can be handled is also determined by the available computational resources.

The solution of the TDSE requires an accurate and efficient generation of the Hamiltonian and electron-field interaction matrix elements. In order to achieve this goal, we approximate the time-dependent wavefunction as

$$
\Psi\left(\boldsymbol{r}_{1}, \ldots, \boldsymbol{r}_{N} ; t\right) \approx \sum_{q} C_{q}(t) \Phi_{q}\left(\boldsymbol{r}_{1}, \ldots, \boldsymbol{r}_{N}\right) .
$$

The $\Phi_{q}\left(\boldsymbol{r}_{1}, \ldots, \boldsymbol{r}_{N}\right)$ are a set of time-independent $N$-electron states formed from appropriately symmetrized products of atomic orbitals. They are expanded as

$\Phi_{q}\left(\boldsymbol{r}_{1}, \ldots, \boldsymbol{r}_{N}\right)=\mathcal{A} \sum_{c, i, j} a_{i j c q} \Theta_{c}\left(x_{1}, \ldots, x_{N-2} ; \hat{\boldsymbol{r}}_{N-1} \sigma_{N-1} ; \hat{\boldsymbol{r}}_{N} \sigma_{N}\right) R_{i}\left(r_{N-1}\right) R_{j}\left(r_{N}\right)$. 
Here $\mathcal{A}$ is the anti-symmetrization operator, the $\Theta_{c}\left(x_{1}, \ldots, x_{N-2} ; \hat{\boldsymbol{r}}_{N-1} \sigma_{N-1} ; \hat{\boldsymbol{r}}_{N} \sigma_{N}\right)$ are channel functions involving the space and spin coordinates $\left(x_{i}\right)$ of $N-2$ core electrons coupled to the angular $(\hat{\boldsymbol{r}})$ and $\operatorname{spin}(\sigma)$ coordinates of the two outer electrons, $R_{i}(r)$ is a radial basis function, and the $a_{i j c q}$ are expansion coefficients. Although resembling a closecoupling ansatz with two continuum electrons, the expansion (4) contains bound states and singly ionized states as well. In general, the atomic oritals, $R_{i}(r)$, are not orthogonal to one another or to the orbitals used to describe the atomic core. If orthogonality constraints are imposed on these functions, additional terms would need to be added to the expansion to relax the constraints. This possibility still exists as an option in our computer code.

In addition to simplifying the expansion, a significant advantage of not forcing such orthogonality conditions is the flexibility gained by being able to tailor the optimization procedures to the individual neutral, ionic, and continuum orbitals. In the BSR code, the outer orbitals (i.e., the $R$ functions above) are expanded in $B$-splines. Factors that depend on angular and spin momenta are separated from the radial degrees of freedom through the construction of the channel functions. Since many Hamiltonian matrix elements share common features, this enables the production of a "formula tape", resulting in an efficient procedure to generate the required matrix elements.

When the expansion (3) is inserted into the Schrödinger equation, we obtain

$$
i \boldsymbol{S} \frac{\partial}{\partial t} \boldsymbol{C}(t)=\left[\boldsymbol{H}_{0}+E(t) \boldsymbol{D}\right] \cdot \boldsymbol{C}(t),
$$

where $\boldsymbol{S}$ is the overlap matrix of the basis functions, $\boldsymbol{H}_{0}$ and $\boldsymbol{D}$ are matrix representations of the field-free Hamiltonian and the dipole coupling matrices, and $\boldsymbol{C}(t)$ is the time-dependent coefficient vector in Eq. (3).

The price to pay for the flexibility in the BSR approach, at least initially, is the representation of the field-free Hamiltonian and the dipole matrices in a non-orthogonal basis. In our previous work [3], we described two methods of combining the BSR method with a highly efficient Arnoldi-Lanczos propagation scheme. In the first one, we diagonalized the overlap matrix $S$ and transformed the problem back to an orthogonal basis before applying the standard propagation scheme [15, 16]. Alternatively, the propagation can be done more directly in the non-orthogonal basis. This only requires a Cholesky decomposition of $\boldsymbol{S}$, but some additional operations at every time step.

A third approach involves a transformation into the eigenbasis by solving the field-free generalized eigenvalue problem first. Details can be found in Guan et al [4]. This method, also used by Laulan and Bachau [17], has the major advantage that the transformation makes it possible to cut unphysically high eigenvalues and the corresponding eigenvectors from the time propagation scheme, thereby making the scheme more stable. At the same time, it simplifies the definition of the initial state and the extraction of the physically interesting information, and it once again allows for the use of the standard Arnoldi-Lanczos time propagation scheme. It was applied in the present work as well. We checked that the results were stable against a variation of both the cut-off energy ( 5.5 a.u. for the results shown below) and the time-step (100 steps per optical cycle).

We first tested our method for the He problem, i.e., two electrons in the presence of a 
bare $\mathrm{He}^{2+}$ core, and will show some example results below. With a box size of 60 a.u. and 81 $B$-splines distributed over this range, with a spacing of 1.0 a.u. near the edge of the box and smaller steps near the origin, the ranks of the field-free Hamiltonian blocks ranged between 10,000 and 20,000. We expect these ranks to increase to 50,000-100,000 for a complex target like Ne. Fortunately, it has become rather straightforward to diagonalize matrices of that size and perform the transformation of the dipole matrices to the eigenbasis on massively parallel computing platforms.

\subsection{Extraction of the cross sections in two-photon double ionization process}

Details regarding the extraction of the fully differential (energies and angles resolved) as well as the total cross section were given by Guan et al [2] and therefore will not be repeated here. To make this paper self-consistent, however, we summarize the most important formulas used for single-color processes and then indicate what has to be done in the case of more than one laser.

In a time-dependent formulation, the dependence of the $N$-photon cross section on the number of photons absorbed not only occurs through the factor $\left(\omega / I_{0}\right)^{N}$, where $\omega$ is the angular frequency and $I_{0}$ is the peak intensity of the laser, but also through the effective interaction time. The result for the latter depends explicitly on the number of photons being absorbed. For two photons it is given by [18]

$$
T_{\text {eff }}^{(N)} \equiv \int_{0}^{\tau} f^{2 N}(t) \mathrm{d} t
$$

with the result

$$
T_{\mathrm{eff}}^{(2)}=35 \tau / 128
$$

for a sine-squared pulse shape of duration of $\tau$.

The energy sharing between the two escaping electrons can be uniquely determined through the hyperangle $\alpha \equiv \tan ^{-1}\left(k_{2} / k_{1}\right)$. We set $E_{1}=E_{\text {exc }} \cos ^{2} \alpha$ and $E_{2}=E_{\text {exc }} \sin ^{2} \alpha$, where $E_{\text {exc }}=2 \omega-I^{2+}$ is the excess energy for two-photon double ionization. For a given energy sharing $\alpha$, the triple-differential cross section (TDCS) can be written as

$\frac{\mathrm{d}^{3} \sigma}{\mathrm{d} \alpha \mathrm{d} \hat{\boldsymbol{k}}_{1} \mathrm{~d} \hat{\boldsymbol{k}}_{2}}=\left(\frac{\omega}{I_{0}}\right)^{2} \frac{1}{T_{\text {eff }}^{(2)}} \int \mathrm{d} k_{1}^{\prime} \mathrm{d} k_{2}^{\prime} k_{1}^{\prime 2} k_{2}^{\prime 2} \delta\left(\alpha-\tan ^{-1}\left(\frac{k_{2}^{\prime}}{k_{1}^{\prime}}\right)\right)\left|\left\langle\Psi_{\boldsymbol{k}_{1}^{\prime}, \boldsymbol{k}_{2}^{\prime}}^{(-)} \mid \Psi(t)\right\rangle\right|^{2}$,

where $|\Psi(t)\rangle$ is the time-propagated wavefunction. Also, the singlet two-electron continuum wavefunction satisfying the incoming-wave boundary condition $(-)$ is given by

$$
\Psi_{\boldsymbol{k}_{1}, \boldsymbol{k}_{2}}^{(-)}\left(\boldsymbol{r}_{1}, \boldsymbol{r}_{2}\right)=\frac{1}{\sqrt{2}}\left[\Phi_{\boldsymbol{k}_{1}}^{(-)}\left(\boldsymbol{r}_{1}\right) \Phi_{\boldsymbol{k}_{2}}^{(-)}\left(\boldsymbol{r}_{2}\right)+\Phi_{\boldsymbol{k}_{2}}^{(-)}\left(\boldsymbol{r}_{1}\right) \Phi_{\boldsymbol{k}_{1}}^{(-)}\left(\boldsymbol{r}_{2}\right)\right]
$$

With the one-electron Coulomb function given by

$$
\Phi_{\boldsymbol{k}}^{(-)}(\boldsymbol{r})=\frac{1}{k} \sum_{l m} \mathrm{i}^{l} e^{-\mathrm{i} \sigma_{l}(k)} \varphi_{k l}^{(c)}(r) Y_{l m}(\hat{\boldsymbol{r}}) Y_{l m}^{*}(\hat{\boldsymbol{k}}),
$$

and the asymptotic behavior

$$
\varphi_{k l}^{(c)}(r) \underset{r \rightarrow \infty}{\longrightarrow} \sqrt{\frac{2}{\pi}} \sin \left(k r+\frac{Z}{k} \ln 2 k r-\frac{l \pi}{2}+\sigma_{l}\right),
$$


with $\sigma_{l}$ as the Coulomb phase, the two-electron Coulomb function (9) is normalized in momentum space according to

$$
\left\langle\Psi_{\boldsymbol{k}_{1}, \boldsymbol{k}_{2}}^{(-)} \mid \Psi_{\boldsymbol{k}_{1}^{\prime}, \boldsymbol{k}_{2}^{\prime}}^{(-)}\right\rangle=\delta\left(\boldsymbol{k}_{1}-\boldsymbol{k}_{1}^{\prime}\right) \delta\left(\boldsymbol{k}_{2}-\boldsymbol{k}_{2}^{\prime}\right)+\delta\left(\boldsymbol{k}_{1}-\boldsymbol{k}_{2}^{\prime}\right) \delta\left(\boldsymbol{k}_{2}-\boldsymbol{k}_{1}^{\prime}\right) .
$$

In the present work, we first generated the one-electron Coulomb functions $\varphi_{k l}^{(c)}(r)$ by employing the routine COULFG of Barnett [19], on the same grid used for the $B$-splines. This choice guarantees a self-consistent grid representation of the time-evolved wavepacket and the final continuum states. We then expressed the function in terms of our $B$-spline basis in order to use the standard integration schemes in that basis.

It is important to remember that there are two indistinguishable electrons in the final channel, and hence the energy sharings described by $\alpha$ and $\pi / 2-\alpha$ represent the same observable event. Therefore, we either need to consider $0 \leq \alpha \leq \pi / 4$ or $\pi / 4 \leq \alpha \leq \pi / 2$ to avoid double counting. The TDCS with respect to the energy of one electron is then given by

$$
\begin{array}{r}
\frac{\mathrm{d}^{3} \sigma}{\mathrm{d} E_{1} \mathrm{~d} \hat{\boldsymbol{k}}_{1} \mathrm{~d} \hat{\boldsymbol{k}}_{2}}=\frac{1}{k_{1} k_{2} \cos ^{2} \alpha}\left(\frac{\omega}{I_{0}}\right)^{2} \frac{1}{T_{\mathrm{eff}}^{(2)}} \int \mathrm{d} k_{1}^{\prime} \mathrm{d} k_{2}^{\prime} k_{1}^{\prime} \delta\left(k_{2}^{\prime}-k_{1}^{\prime} \tan \alpha\right) \\
\times\left|\sum_{L=0,2, l_{1} l_{2}} \chi_{l_{1} l_{2}}\left(k_{1}^{\prime}, k_{2}^{\prime}\right) \mathcal{Y}_{l_{1} l_{2}}^{L M}\left(\hat{\boldsymbol{k}}_{1}, \hat{\boldsymbol{k}}_{2}\right) \mathcal{F}_{l_{1} l_{2}}^{L}\left(k_{1}^{\prime}, k_{2}^{\prime}\right)\right|^{2} .
\end{array}
$$

Here we have defined the two-electron projection

$$
\mathcal{F}_{l_{1} l_{2}}^{L}\left(k_{1}, k_{2}\right) \equiv\left\langle\Psi_{k_{1} l_{1}, k_{2} l_{2}}^{L(-)} \mid \Psi(t)\right\rangle,
$$

with the phase factor

$$
\chi_{l_{1} l_{2}}\left(k_{1}, k_{2}\right)=(-\mathbf{i})^{l_{1}+l_{2}} e^{\mathrm{i}\left(\sigma_{l_{1}}\left(k_{1}\right)+\sigma_{l_{2}}\left(k_{2}\right)\right)},
$$

and

$$
\Psi_{k_{1} l_{1}, k_{2} l_{2}}^{L(-)} \equiv \mathcal{A}\left\{\varphi_{k_{1} l_{1}}^{(c)}\left(r_{1}\right) \varphi_{k_{2} l_{2}}^{(c)}\left(r_{2}\right) \mathcal{Y}_{l_{1} l_{2}}^{L}\left(\hat{\boldsymbol{k}}_{1}, \hat{\boldsymbol{k}}_{2}\right)\right\}
$$

as the properly antisymmetrized product of two one-electron radial Coulomb functions and coupled spherical harmonics $\mathcal{Y}_{l_{1} l_{2}}^{L}$ for the angular part. [Since $M=0$ for linearly polarized radiation, we omit it to simplify the notation.]

By collecting all ionization events, the total cross section for two-photon double ionization is obtained as

$\sigma_{\mathrm{NS}}=\int \mathrm{d} \alpha \int \mathrm{d} \hat{\boldsymbol{k}}_{1} \mathrm{~d} \hat{\boldsymbol{k}}_{2} \frac{\mathrm{d}^{3} \sigma}{\mathrm{d} \alpha \mathrm{d} \hat{\boldsymbol{k}}_{1} \mathrm{~d} \hat{\boldsymbol{k}}_{2}}=\left(\frac{\omega}{I_{0}}\right)^{2} \frac{1}{T_{\text {eff }}^{(2)}} \int \mathrm{d} k_{1} \mathrm{~d} k_{2} \frac{\mathrm{d}^{2} \mathcal{P}\left(k_{1}, k_{2}\right)}{\mathrm{d} k_{1} \mathrm{~d} k_{2}}$,

where we have introduced the momentum distribution

$$
\frac{\mathrm{d}^{2} \mathcal{P}\left(k_{1}, k_{2}\right)}{\mathrm{d} k_{1} \mathrm{~d} k_{2}}=\sum_{L=0,2} \sum_{l_{1} l_{2}}\left|\mathcal{F}_{l_{1} l_{2}}^{L}\left(k_{1}, k_{2}\right)\right|^{2} .
$$

Similarly, the momentum distribution is given by

$$
\frac{\mathrm{d}^{2} \mathcal{E}\left(E_{1}, E_{2}\right)}{\mathrm{d} E_{1} \mathrm{~d} E_{2}}=\frac{1}{k_{1} k_{2}} \frac{\mathrm{d}^{2} \mathcal{P}\left(k_{1}, k_{2}\right)}{\mathrm{d} k_{1} \mathrm{~d} k_{2}} .
$$

Below we will also show results obtained for two linearly polarized XUX laser pulses, for which the electric field is given by

$$
\boldsymbol{E}(t)=\left[E_{1} f_{1}(t) \sin \left(\omega_{1} t\right)+E_{2} f_{2}\left(t-T_{d}\right) \sin \left(\omega_{2}\left(t-T_{d}\right)\right)\right] \boldsymbol{e}_{z} .
$$


Here $\omega_{1}$ and $\omega_{2}$ are the two central frequencies while $T_{d}$ is the time delay between the two pulses. The total time duration can be written as $\tau=\max \left\{\tau_{1}, \tau_{2}+T_{d}\right\}$.

Note that this time duration depends on the durations of pulse 1 , pulse 2 , and the time delay between them. Hence, the definition of an "effective interaction time" is by no means straightforward and even a "generalized cross section" (which should be independent of these parameters) cannot be defined in the standard sense. Momentum distributions, however, can be obtained by calculating and then squaring the magnitude of the function (14). Energy distributions for the He case were recently reported by Foumouo et al [20].

\section{RESULTS AND DISCUSSION}

As a first test of our newly developed approach, we applied it to the single-color two-photon double ionization of helium, for which a variety of benchmark data exist for comparison (see, for example, refs. [1, 2] and references therein). In this particular test, we confined the system to a spatial box of $r_{\max }=60$ a.u. We used $81 B$-splines of order 8 to span this configuration space, with the knot sequence chosen in such a way that the spatial variation of the wavefunction close to the nucleus (determined by the nuclear charge $Z$ ) and far away from the nucleus (determined by the highest energy of a free electron) could be represented accurately.

Having obtained the initial state of the system by diagonalizing the field-free Hamiltonian of the ${ }^{1} \mathrm{~S}^{\mathrm{e}}$ symmetry and taking the eigenvector to the lowest eigenvalue $(-2.90330$ a.u. in our particular case), the time propagation in the laser field is accomplished through the ArnoldiLanczos algorithm. Compared to other time-propagation approaches, such as leapfrog or a split-operator approach [21], the present scheme allows us to take relatively large steps in time. Specifically, using only 100 steps per optical cycle (o.c.) is sufficient to achieve converged solutions of the TDSE for the cases presented in this paper.

The results presented below were obtained with a sine-squared pulse with a peak intensity of $5 \times 10^{14} \mathrm{~W} / \mathrm{m}^{2}$ and a time duration of 10 optical cycles. For this case, we first checked the total cross sections for photon energies of 42 and $50 \mathrm{eV}$, respectively. These values were chosen to ensure that the box size and the length of the laser pulse were suitable to properly define a generalized cross section for two-photon double ionization. The present results of $4.02 \times 10^{-53} \mathrm{~cm}^{4} \mathrm{~s}$ and $2.20 \times 10^{-52} \mathrm{~cm}^{4} \mathrm{~s}$ agree in a satisfactory way with the corresponding values of $3.76 \times 10^{-53} \mathrm{~cm}^{4} \mathrm{~s}$ and $1.89 \times 10^{-52} \mathrm{~cm}^{4} \mathrm{~s}$ obtained in an entirely different finiteelement discrete-variable representation (FE-DVR) [2].

\subsection{Triple-differential cross sections for one-color two-photon double ionization}

Figure 1 displays the triple-differential cross section for two-photon double ionization of helium in a 10-cycle sine-squared laser pulse of central photon energy $42 \mathrm{eV}$ and peak intensity $5 \times 10^{14} \mathrm{~W} / \mathrm{cm}^{2}$ for equal energy sharing $\left(E_{1}=E_{2}=2.5 \mathrm{eV}\right)$ of the two outgoing electrons. These results are for the coplanar geometry, where the electric field vector of the linearly polarized laser field and the momentum vectors of the two escaping electrons all lie in the same plane. 

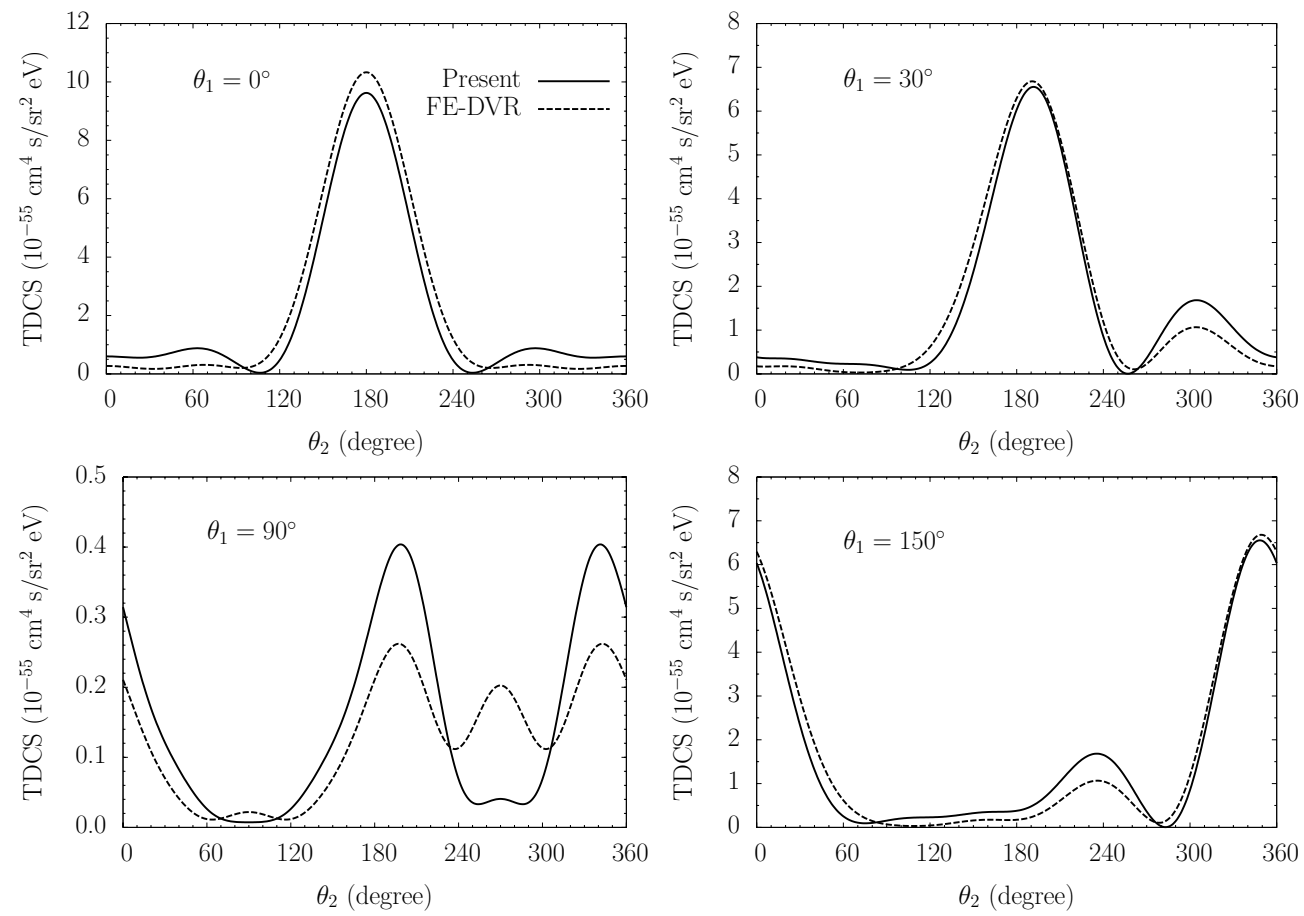

Figure 1. The coplanar triple-differential cross section for two-photon double ionization of helium in a 10-cycle sine-squared laser pulse of central photon energy $42 \mathrm{eV}$ and peak intensity $5 \times 10^{14} \mathrm{~W} / \mathrm{cm}^{2}$ for equal energy sharing $\left(E_{1}=E_{2}=2.5 \mathrm{eV}\right)$ of the two outgoing electrons. The angle listed in the figure is the angle between the laser polarization vector and one of the two escaping electrons, while the emission angle of the second electron varies. The present TDBSR results are compared with those from our previous FE-DVR calculation [2].

Given the very different implementations of the current TDBSR method and the FEDVR approach, we see satisfactory agreement between the present results and those obtained earlier. Note that these results were obtained with $\left(L_{\max }, l_{1, \max }, l_{2, \max }\right)=(3,3,3)$. We are aware of potential convergence problems with these parameters, especially for kinematic situations where the cross section is small (e.g., at $\theta_{1}=90^{\circ}$ ). However, since the principal motivation for performing these particular calculations was to test the method and the accompanying computer code, we consider the test successful and hence move on to the two-color-case in the next subsection.

\subsection{Momentum and energy distributions for two-color two-photon double ionization}

We now consider the process of double photoionization by absorption of two photons at different central photon energies. In other words, the target helium atom is exposed to the irradiation by two laser pulses, of potentially different frequencies and with a controllable time delay. This allows us to study the mechanism of the breakup problem by applying pulses of various durations for each pulse and also modifying the time delay between them. The following two-color laser parameters were considered in this work: pulse 1 has a central photon energy of $35.3 \mathrm{eV}$, a time duration of 12 o.c., and a peak intensity of $1 \times 10^{14} \mathrm{~W} / \mathrm{cm}^{2}$, while the corresponding parameters for pulse 2 are $57.1 \mathrm{eV}, 14$ o.c., and $1 \times 10^{13} \mathrm{~W} / \mathrm{cm}^{2}$, 

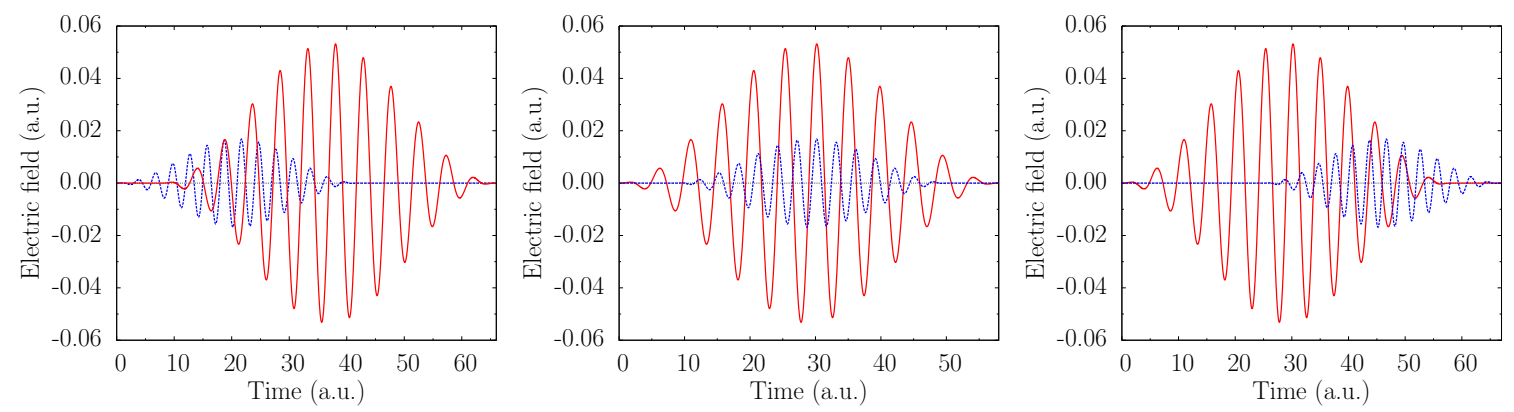

Figure 2. Electric field of the two-color laser pulse. The laser parameters are: $\omega_{1}=35.3 \mathrm{eV}$, $\tau_{1}=12$ o.c. at a peak intensity of $10^{14} \mathrm{~W} / \mathrm{cm}^{2}$ (solid curve), and $\omega_{2}=57.1 \mathrm{eV}, \tau_{2}=14$ o.c. at a peak intensity of $10^{13} \mathrm{~W} / \mathrm{cm}^{2}$ (dashed curve). The time delays between the maxima of the two pulses are, from left to right, $-16.5,0.0$, and 16.5 a.u.

respectively. A similar two-color laser pulse was studied in the recent work by Foumouo et al [20].

In the present work, we are interested in the mechanism for the ejection of two electrons when the time delay between the two pulses is varied. Figure 2 displays the time-dependent electric fields of the two-color pulses for time delays of -16.5 a.u. (i.e., pulse 2 comes first), no delay (both pulses come simultaneously, and a delay of +16.5 a.u. (pulse 1 comes first). Note that +16.5 a.u correspond to approximately 400 attoseconds.

Figure 3 depicts the momentum and energy distributions of the two escaping electrons for the above two-color laser pulses. For the negative time delay with pulse 2 arriving first, we observe a strong peak around $E_{1}=E_{2} \simeq 6.7 \mathrm{eV}$, as well as two weaker peaks around $\left(E_{1}, E_{2}\right) \simeq(2.7,32) \mathrm{eV}$ and $\left(E_{1}, E_{2}\right) \simeq(32,2.7) \mathrm{eV}$, respectively. This suggest that the target electrons predominantly absorb the two photons in the highly correlated way similar to the nonsequential double ionization mechanism, even though the photon energies are different. Recent calculations by Feist et al [22], using a single-color laser, also demonstrate that the conventional scenario of "direct" vs "sequential" two-photon double ionization breaks down for attosecond XUV pulses, even when the sequential process is energetically allowed.

In the above two-color case, the two ejected electrons share the excess energy of $E_{\text {exc }}=\omega_{1}+\omega_{2}-I^{2+} \simeq 13.5 \mathrm{eV}$ almost equally. However, there is apparently another ionization channel open for the present laser parameters. Since the target electrons interact with pulse 2 of energy $57.1 \mathrm{eV}$ first, the first electron is ejected with a kinetic energy of about $32(\simeq 57.1-24.6) \mathrm{eV}$ before the second electron is ionized with an energy of $2.7(\simeq 57.1-54.4) \mathrm{eV}$ from the ground state of the residual $\mathrm{He}^{+}(1 \mathrm{~s})$ ion. This channel is characteristic of sequential ionization by two photons of the same high frequency and very different from the direct channel. In fact, in this case there is no electron left for the second pulse to interact with.

To improve the visibility of the remaining figures, we will show the energy distributions only in the energy region below $20 \mathrm{eV}$ for the cases of zero and positive time delays. When there is no time delay between the two maxima of the electric fields, the principal structure observed is still around $E_{1}=E_{2} \simeq 6.7 \mathrm{eV}$ in the energy distribution. The mechanism in this 

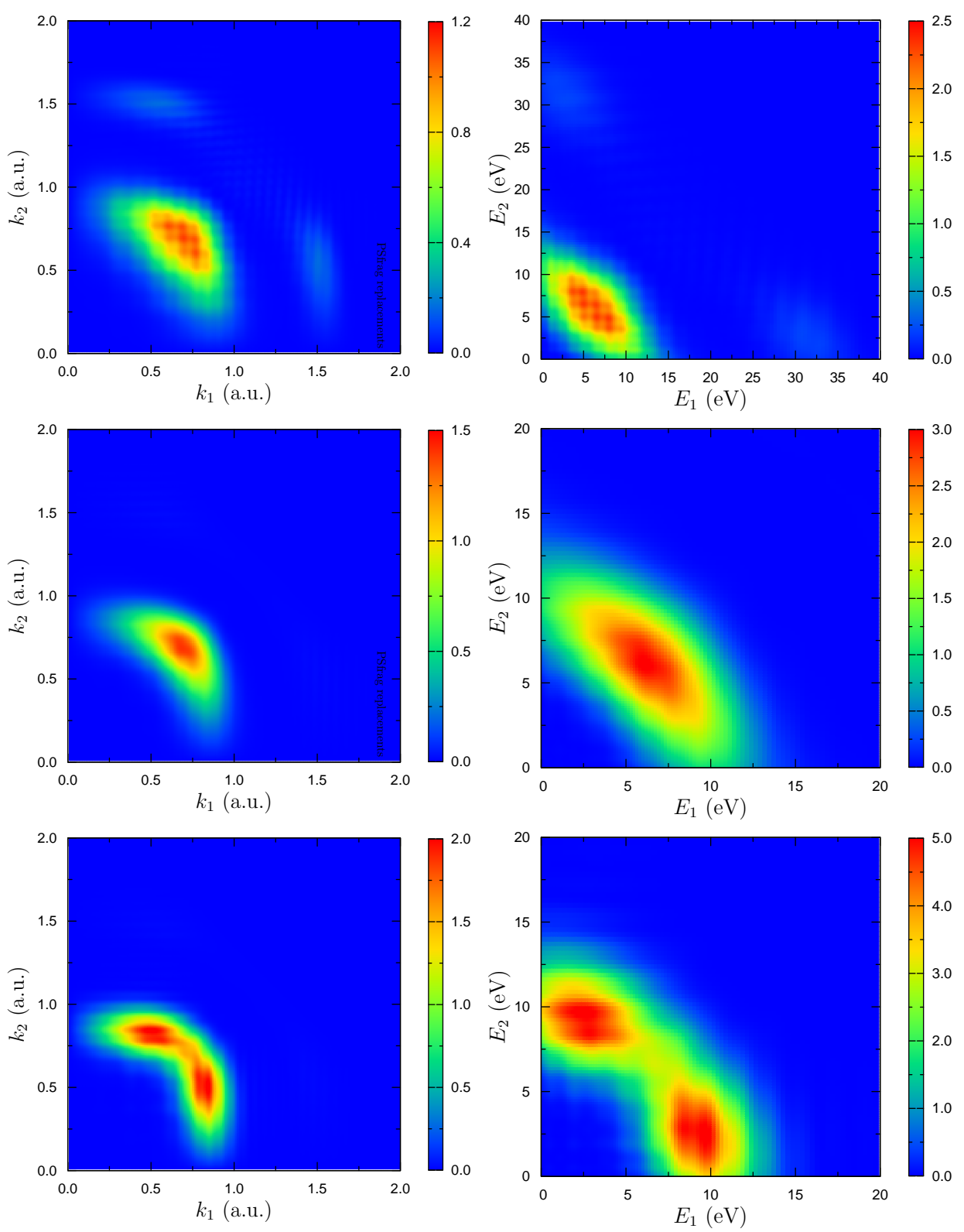

Figure 3. Momentum (left column) and energy (right column) distributions of the two escaping electrons in a two-color laser pulse. The laser parameters are the same as in figure 2 The color bars for the first row (negative time delay) correspond to units of $10^{-5}$ a.u., while the other two rows are given in units of $10^{-4}$ a.u. The time delays between the two laser pulses, from top to bottom, are $-16.5,0.0$, and +16.5 a.u., respectively.

case is thus similar to what we observed for the negative time delay.

On the other hand, when the time delay is increased further, for example up to +16.5 a.u., the two electrons are ejected a very different way. In this case, the main structure revealed from figure 3 exhibits two peaks at $\left(E_{1}, E_{2}\right) \simeq(10.0,2.5) \mathrm{eV}$ and $(2.5,10.0) \mathrm{eV}$. This is due to the fact that pulse 1 comes first and hence, after absorption of one photon of frequency $\left(\omega_{1}\right)$ one electron with the kinetic energy of $10.7(=35.3-24.6) \mathrm{eV}$ is ejected. The time delay of +16.5 a.u. is sufficiently long for the residual $\mathrm{He}^{+}$ion to relax into its ground state after 

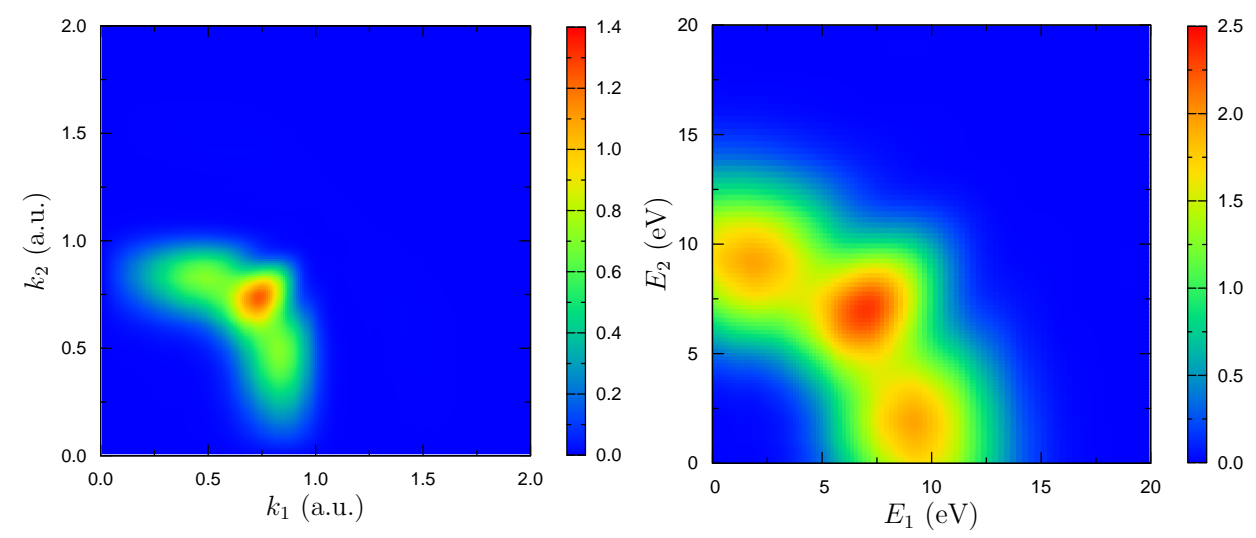

Figure 4. Momentum (left) and energy (right) distributions of the two escaping electrons in a two-color laser pulse. The wavelengths and laser intensities are the same as in figure 2 , but in this case the pulselengths are only 10 optical cycles with a delay of 15 a.u. The color bars are given in units of $10^{-4}$ a.u.

the single ionization. When this process is followed by the absorption of the second photon of $57.1 \mathrm{eV}\left(\omega_{2}\right)$, the other electron carries away the excess energy of $2.7=(57.1-54.4) \mathrm{eV}$. This suggests that the two electrons are basically removed through a sequential process.

We then explored the parameter space in more detail by varying both the pulselength and the delay. An even more striking example of the competing processes is exhibited in figure 4. For the same wavelengths and intensities as before, but with a relatively small change in the pulselengths to 10 optical cycles and a delay of 15 a.u. ( $\approx 360$ attoseconds), sequential and non-sequential absorption of the two photons play an almost equally important role in the double ionization process.

From the discussion and examples given above, it is clear that the time delay plays a decisive role in determining how the two electrons are ejected by two-color XUV laser pulses. Depending on the details of time delay, the electrons can be ejected in ways either similar to the sequential or the non-sequential process. Our findings qualitatively agree with those of Foumouo et al [20] for the two-color problem and Feist et al [22] in the single-color problem. They serve as an independent confirmation of their predictions, and also provide us confidence in our newly developed computer code.

\section{CONCLUSIONS}

We have presented a general method to calculate two-photon double ionization of atoms using a $B$-spline $R$-matrix approach in connection with an efficient Arnoldi-Lanczos time propagation scheme. Test calculations for helium revealed good agreement with previous benchmark results obtained with different and entirely independent methods. The method was then applied to two-photon double ionization of helium using two time-delayed XUV laser pulses. The latter results confirm that attosecond spectroscopy will provide a "microscope" to examine and also control the way electrons interact in atomic and molecular targets.

We are currently in the process of generating and then transforming the corresponding 
matrices for the two-photon double ionization problem of neon atoms. This will allow us to make a direct comparison with the recent experiments of Moshammer et al [23] carried out at the FLASH facility in Hamburg. While the additional complication of a residual core with non-zero angular momentum is substantial, the current method has been formulated in such a way that these calculations are effectively limited by the available hardware (i.e. supercomputer facilities) rather than special-purpose software. As long as the field-free and the dipole matrices can first be generated, in any primitive basis, and then transformed to the eigenbasis, the time propagation essentially becomes a matter of fast matrix-vector multiplications, for which highly tuned subroutines are available.

Consequently, we are confident that we will be able to generate results for complex targets in the very near future. In addition, we plan to adapt the method to simple linear molecules that are amenable to single-center expansion techniques.

\section{Acknowledgments}

This work was supported by the United States National Science Foundation under grants No. PHY-0757755 (XG and KB) and PHY-0555226 (CJN, OZ, and KB). We gratefully acknowledge supercomputer resources provided by the U. S. Department of Energy through its National Energy Research Scientific Computer Center (NERSC) and the NSF through Teragrid allocations under TG-PHY090031.

\section{References}

[1] J. Feist, S. Nagele, R. Pazourek, E. Persson, B. I. Schneider, L.A. Collins, and J. Burgdörfer, Phys. Rev. A 77 (2008) 043420.

[2] X. Guan, K. Bartschat, and B. I. Schneider, Phys. Rev. A 77 (2008) 043421.

[3] X. Guan, O. Zatsarinny, K. Bartschat, B. I. Schneider, J. Feist, and C. J. Noble, Phys. Rev. A 76 (2007) 053411.

[4] X. Guan, C. J. Noble, O. Zatsarinny, K. Bartschat, and B. I. Schneider, Phys. Rev. A 77 (2008) 043421.

[5] O. Zatsarinny, Comp. Phys. Commun. 174 (2006) 273.

[6] X. Guan, C. J. Noble, O. Zatsarinny, K. Bartschat, and B. I. Schneider, submitted to Comp. Phys. Commun. (2008).

[7] P. G. Burke, P. Francken, and C. J. Joachain, J. Phys. B 24 (1991) 761.

[8] M. Plummer and C. J. Noble, J. Phys. B 36 (2003) L219.

[9] P. G. Burke and V. M. Burke, J. Phys. B 30 (1997) L383.

[10] H. W. van der Hart, M. A. Lysaght, and P. G. Burke, Phys. Rev. A 76 (2007) 043405.

[11] M. A. Lysaght, H. W. van der Hart, and P. G. Burke, Phys. Rev. Lett. 101 (2008) 253001.

[12] S. X. Hu and L. A. Collins, Phys. Rev. A 73 (2006) 023405.

[13] S. X. Hu and L. A. Collins, Phys. Rev. Lett. 94 (2005) 073004.

[14] K. T. Taylor, J. S. Parker, D. Dundas, K. J. Meharg, L.-Y. Peng, B. J. S. Doherty, and J. F. McCann, Physica Scripta T110 (2004) 154.

[15] T. J. Park and J. C. Light, J. Chem. Phys. 85 (1986) 5870.

[16] B. I. Schneider and L. A. Collins, J. Non-Cryst. Solids 351 (2005) 1551.

[17] S. Laulan and H. Bachau, Phys. Rev. A 68 (2003) 013409.

[18] E. Foumouo, G. L. Kamta, G. Edah, and B. Piraux, Phys. Rev. A 74 (2006) 063409. 
[19] A. R. Barnett, in Computational Atomic Physics, ed. K. Bartschat (Springer, Heidelberg and New York, 1996).

[20] E. Foumouo, P. Antoine, H. Bachau, and B. Pireaux, New J. Phys. 10 (2008) 025017.

[21] S. X. Hu, J. Colgan, and L. A. Collins, J. Phys. B 38 (2005) L35.

[22] J. Feist, S. Nagele, R. Pazourek, E. Persson, B. I. Schneider, L. A. Collins, and J. Burgdörfer, arXiv:0812.0373, submitted to Phys. Rev. Lett. (2009).

[23] R. Moshammer, Y. H. Jiang, L. Foucar, A. Rudenko, Th. Ergler, C. D. Schröter, S. Lüdemann, K. Zrost, D. Fischer, J. Titze, T. Jahnke, M. Schöffler, T. Weber, R. Dörner, T. J. M. Zouros, A. Dorn, T. Ferger, K. U. Kühnel, S. Düsterer, R. Treusch, P. Radcliffe, E. Plönjes, and J. Ullrich, Phys. Rev. Lett. 98 (2007) 203001. 